\title{
Experimental Comparison of Methods for Multivariable Frequency Response Function Estimation
}

Erik Wernholt, Stig Moberg

Division of Automatic Control

E-mail: erikw@isy.liu.se, stig@isy.liu.se

29th October 2007

Report no.: LiTH-ISY-R-2827

Submitted to 17th IFAC World Congress, Seoul, Korea

Address:

Department of Electrical Engineering

Linköpings universitet

SE-581 83 Linköping, Sweden

WWW: http://www. control.isy.liu.se

AUTOMATIC CONTROL

REGLERTEKNIK

LINKÖPINGS UNIVERSITET

Technical reports from the Automatic Control group in Linköping are available from http://www. control.isy.liu.se/publications. 


\begin{abstract}
Nonparametric estimation methods for the multivariable frequency response function are experimentally evaluated using closed-loop data from an industrial robot. Three classical estimators $\left(\mathrm{H}_{1}\right.$, joint input-output, arithmetic mean) and two estimators based on nonlinear averaging techniques (harmonic mean, geometric/logarithmic mean) are considered. The estimators based on nonlinear averaging give the best results, followed by the arithmetic mean estimator, which gives a slightly larger bias. The joint input-output estimator, which is asymptotically unbiased in theory, turns out to give large bias errors for low frequencies. Finally, the $\mathrm{H}_{1}$ estimator gives the largest bias for all frequencies.
\end{abstract}

Keywords: System identification, frequency response methods, multivariable systems, non-parametric identification, closed-loop identification, industrial robots 


\section{Introduction}

Nonparametric estimates of the frequency response function (FRF) give valuable information about the dynamics of a system and are often used as an intermediate step in a parametric identification process to assess the quality of the measurements and visualize the complexity of the modeling problem. In a second step, a parametric model can be estimated, either by, 1) treating the estimated nonparametric FRF as a measurement and minimize the discrepancy between the model and the estimated FRF as is done in, e.g., experimental modal analysis (Verboven, 2002), or, 2) directly from the measured input and output data, (Ljung, 1999; Pintelon and Schoukens, 2001).

This paper studies five different estimators: $H_{1}$, joint input-output (JIO), arithmetic mean (ARI), logarithmic mean ( $L O G)$, and harmonic mean (HAR). These estimators will be described in more detail in the next section. The experimental comparison of the estimators will be carried out using measurements from the first three axes of an industrial robot in the ABB IRB6600 series, see Figure 1. The robot application is interesting since it gives many challenging problems for system identification methods. The robot dynamics is multivariable, nonlinear, oscillatory, and, moreover, data must be collected in closed loop. Examples when nonparametric FRF estimates are used for the identification of parametric robot models can be found in, e.g., Wernholt and Moberg (2007), Öhr et al. (2006), Berglund and Hovland (2000), and Khorrami et al. (1995).

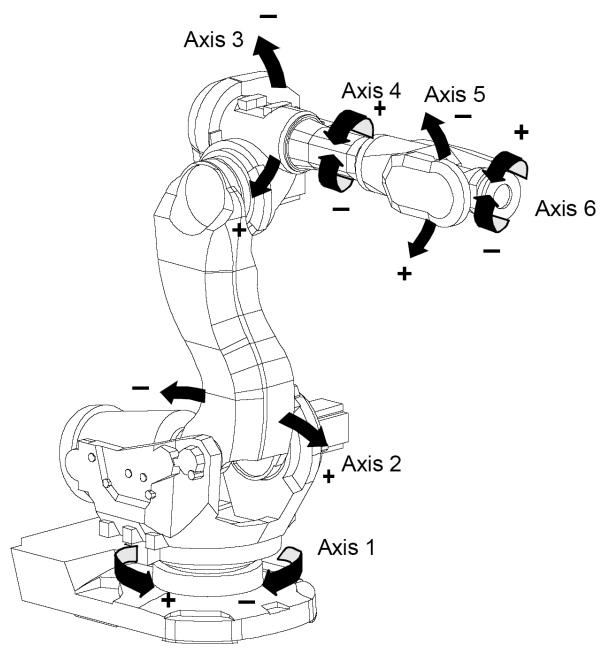

Figure 1: The ABB manipulator IRB6600.

The five estimators differ in requirements on the measurement setup and signal-to-noise ratio (SNR), as well as in bias and variance properties. Using nonparametric frequency-domain methods for closed-loop data might give biased estimates. The JIO estimator is asymptotically unbiased, and the bias of the other estimators varies depending on the SNR. A recent study on the first three estimators can be found in Wernholt and Gunnarsson (2007). See also Pintelon and Schoukens (2001), Guillaume (1998), and Verboven (2002). 


\section{Measurement Setup}

The open-loop system to be identified is unstable, which makes it necessary to collect data while the robot controller is running in closed loop. Consider therefore the setup in Figure 2, where the controller takes as input the difference between the reference signal $r$ and the measured and sampled output $y$, and $u$ is the input. The disturbance $v$ contains various sources of noise and disturbances.

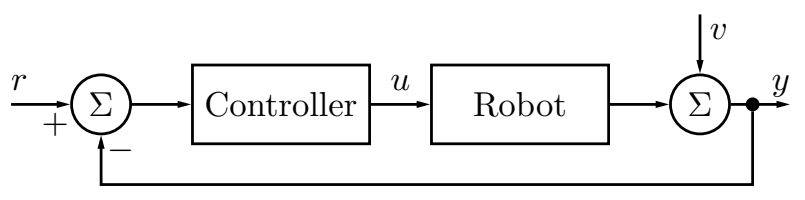

Figure 2: Closed loop measurement setup.

Remark 1 Figure 2 is a simplified block diagram since some disturbances enter early in the robot system, such as motor torque ripple (Gutt et al., 1996).

An experimental control system is used, which enables the use of off-line computed reference signals for each motor controller. The experiments are performed by first moving the robot arm into a position and then applying a speed reference signal to the robot controller. The resulting motor torques (actually the torque reference to the torque controller) and angular positions are sampled and stored. The measured angular positions are then filtered and differentiated to obtain estimates of the motor angular speeds, which are here considered as the output signals.

To avoid leakage effects in the discrete Fourier transform (DFT), which is used by the estimation methods, the excitation signal, $r$, is assumed to be $N_{P^{-}}$ periodic, $r\left(t+N_{P} T_{s}\right)=r(t)$ with $T_{s}$ the sampling period, and an integer number of periods of the steady-state response are collected.

\section{$3 \quad$ FRF Estimation Methods}

\subsection{Basic Idea}

Consider the DFTs of the input, $U\left(\omega_{k}\right)$, output, $Y\left(\omega_{k}\right)$, and reference, $R\left(\omega_{k}\right)$, for the $N_{P}$ frequencies $\omega_{k}=k \frac{2 \pi}{N_{P} T_{s}}, k=1,2, \ldots, N_{P}$. Assuming a linear system and periodic data, the following mapping holds exactly in the noise-free case

$$
Y\left(\omega_{k}\right)=G\left(\omega_{k}\right) U\left(\omega_{k}\right)
$$

where $G\left(\omega_{k}\right) \in \mathbb{C}^{n_{y} \times n_{u}}$ is the FRF of the linear system. To be able to estimate $G\left(\omega_{k}\right)$ from data, at least $n_{u}$ different experiments are needed. The data vectors from $n_{e} \geq n_{u}$ different experiments are collected into matrices (bold-face in the sequel) where each column corresponds to one experiment. The input-output relation can then be written as

$$
\mathbf{Y}\left(\omega_{k}\right)=G\left(\omega_{k}\right) \mathbf{U}\left(\omega_{k}\right)
$$


where $\mathbf{U}\left(\omega_{k}\right) \in \mathbb{C}^{n_{u} \times n_{e}}$ and $\mathbf{Y}\left(\omega_{k}\right) \in \mathbb{C}^{n_{y} \times n_{e}}$. If $\mathbf{U}\left(\omega_{k}\right)$ has rank $n_{u}$, an estimate of $G\left(\omega_{k}\right)$ can be formed by, e.g., using the $H_{1}$ estimator (Guillaume et al., 1996; Pintelon and Schoukens, 2001; Verboven, 2002)

$$
\widehat{G}^{\mathrm{H}_{1}}\left(\omega_{k}\right)=\mathbf{Y}\left(\omega_{k}\right) \mathbf{U}^{H}\left(\omega_{k}\right)\left[\mathbf{U}\left(\omega_{k}\right) \mathbf{U}^{H}\left(\omega_{k}\right)\right]^{-1},
$$

where $(\cdot)^{H}$ denotes complex conjugate transpose.

Disturbances as well as nonlinearities will modify the mapping (1), which in turn will introduce errors in the estimated FRF. For a nonlinear system, we are looking for a linear approximation of the input-output behavior. The properties of such approximations have been extensively studied in, e.g., Schoukens et al. (2005) and Dobrowiecki and Schoukens (2007). See also Wernholt and Gunnarsson (2006) for some results on how nonlinearities affect the FRF estimation of industrial robots.

\subsection{Some Classical Estimators}

For the single input, single output (SISO) case, a number of different nonparametric FRF estimators have been suggested in the literature (see, e.g., Guillaume et al., 1992; Pintelon and Schoukens, 2001; Wellstead, 1981), which all have different properties regarding bias and variance. These estimators can often be generalized to the multiple input, multiple output (MIMO) case, as for the $\mathrm{H}_{1}$ estimator (3).

If the reference signal is measured, an asymptotically $\left(n_{e} \rightarrow \infty\right)$ unbiased estimator has been proposed in Wellstead (1981), which can be generalized to the MIMO case as (Cobb and Mitchell, 1990; Verboven, 2002)

$$
\widehat{G}^{\mathrm{JIO}}=\mathbf{Y} \mathbf{R}^{H}\left[\mathbf{U} \mathbf{R}^{H}\right]^{-1}
$$

where JIO stands for joint input-output estimator (cf. Ljung, 1999, p. 438). (For notational simplicity, the frequency argument will be omitted when not explicitly needed.) This estimator is based on the instrumental variables principle and is also called $H_{\mathrm{IV}}, H_{s}, H_{c}$, or 3 -channel FRF estimator in the literature (Cobb and Mitchell, 1990; Verboven, 2002).

In this paper, we will assume that $n_{e}$ is a multiple of $n_{u}$ such that the DFT matrices can be partitioned into $M$ blocks of size $n_{u} \times n_{u}$ as

$$
\mathbf{R}\left(\omega_{k}\right)=\left[\begin{array}{lll}
\mathbf{R}^{[1]}\left(\omega_{k}\right) & \ldots & \mathbf{R}^{[M]}\left(\omega_{k}\right)
\end{array}\right]
$$

and similarly for $\mathbf{U}\left(\omega_{k}\right)$ and $\mathbf{Y}\left(\omega_{k}\right)$. If the same excitation is used in all blocks with synchronized measurements (e.g., by using multiple periods and treating each period as a "block"), then the JIO estimator reduces to the errors-invariables $^{1}$ (EIV) estimator (Guillaume et al., 1996; Pintelon and Schoukens, 2001)

$$
\widehat{G}^{\mathrm{EIV}}=\left[\frac{1}{M} \sum_{m=1}^{M} \mathbf{Y}^{[m]}\right]\left[\frac{1}{M} \sum_{m=1}^{M} \mathbf{U}^{[m]}\right]^{-1} .
$$

The EIV estimator does not require the reference signal to be known and this is also the maximum likelihood estimator for Normal distributed noise (Guillaume

\footnotetext{
${ }^{1}$ Measurements $Y=G U+V_{y}$ and $U+V_{u}$ with measurement noise $V_{u}$ and $V_{y}$.
} 
et al., 1996). The estimator is therefore often regarded as the optimal one to use, given that the measurements are synchronized and the same excitation is used in all blocks.

Another useful estimator is the arithmetic mean (ARI) estimator (Guillaume, 1998; Pintelon and Schoukens, 2001)

$$
\widehat{G}^{\mathrm{ARI}}=\frac{1}{M} \sum_{m=1}^{M} \widehat{G}^{[m]},
$$

where

$$
\widehat{G}^{[m]}=\mathbf{Y}^{[m]}\left[\mathbf{U}^{[m]}\right]^{-1} .
$$

In case of measurement noise on the input, or closed-loop data, this estimator gives less bias than the $\mathrm{H}_{1}$ estimator (Pintelon and Schoukens, 2001).

\subsection{Estimators Based on Nonlinear Averaging Techniques}

The averaging in (7) can be generalized to include nonlinear averaging techniques. This has been studied in Guillaume et al. (1992) for SISO systems and Guillaume (1998) extends some of these results to MIMO systems. The FRF is then estimated as

$$
\widehat{G}=g^{-1}\left(\frac{1}{M} \sum_{m=1}^{M} g\left(\widehat{G}^{[m]}\right)\right),
$$

where $z=g(x)$ is some nonlinear function with inverse $x=g^{-1}(z)$. Here, we will consider the following functions

$$
g(x)= \begin{cases}x & \text { arithmetic mean } \\ \log x & \text { logarithmic mean } \\ x^{-1} & \text { harmonic mean }\end{cases}
$$

In Guillaume et al. (1992), the properties of FRF estimators based on nonlinear averaging are studied and compared with the classical estimators. They include analytical bias expressions for a number of different estimators when the measurement noise in the input and output signals are uncorrelated (i.e., not a closed-loop setup). Their conclusion is that the use of many classical estimators, such as $\mathrm{H}_{1}$, is not advisable in most practical situations due to larger bias errors. For non-synchronous measurements (where EIV is not applicable), they propose to use estimators based on nonlinear averaging, where the logarithmic mean estimator (also called geometric mean) performs well in all their analyzed situations. This estimator is also particularly robust to outliers.

Using the complex logarithm, $\log x=\log |x|+j \arg x$, will introduce some problems when averaging the phase, due to the phase wrapping at $-\pi(-\pi<$ $\arg x \leq \pi$ ). A solution to this problem is given in Guillaume et al. (1992) for the scalar case by multiplying each $\widehat{G}^{[m]}\left(\omega_{k}\right)$ by $e^{-j \varphi\left(\omega_{k}\right)}$ before the averaging, and afterward multiply the result by $e^{j \varphi\left(\omega_{k}\right)}$, where the phases $\varphi\left(\omega_{k}\right)$ are obtained using some other estimator. The observations are then close to the positive real axis when performing the averaging.

For multivariable systems, the nonlinear averaging functions can either be applied element-wise or by treating them as matrix functions. The element-wise 
approach gives worse estimates, which has been showed in Guillaume (1998) and also seen in our experimental results. The matrix function approach $g(A)$, where $A \in \mathbb{C}^{n \times n}$, can be described by using an eigenvalue decomposition

$$
A=V \Lambda V^{-1}
$$

where $V$ contains the $n$ orthogonal (if $A=A^{T}$ ) eigenvectors and $\Lambda$ is a diagonal matrix, $\Lambda=\operatorname{diag}\left\{\lambda_{l}\right\}_{l=1}^{n}$, with the $n$ eigenvalues. A matrix function $g(A)$ is then given by

$$
g(A)=V g(\Lambda) V^{-1}=V \operatorname{diag}\left\{g\left(\lambda_{l}\right)\right\}_{l=1}^{n} V^{-1} .
$$

Using this, the logarithmic mean (LOG) estimator is finally given by

$$
\widehat{G}^{\mathrm{LOG}}=P_{1}^{-1} \exp \left(\frac{1}{M} \sum_{m=1}^{M} \log \left(P_{1} \widehat{G}^{[m]}\right)\right),
$$

where exp and $\log$ are treated as matrix functions (9). The matrix $P_{1}$ is used to avoid the phase wrapping problems. Here, the matrix is selected as

$$
P_{1}=V^{[1]} \operatorname{diag}\left\{e^{-j \arg \lambda_{l}^{[1]}}\right\}_{l=1}^{n}\left[V^{[1]}\right]^{-1},
$$

where $\widehat{G}^{[1]}=V^{[1]} \Lambda^{[1]}\left[V^{[1]}\right]^{-1}$ (see Guillaume, 1998).

The last estimator to consider, the harmonic mean (HAR) estimator, is given by

$$
\widehat{G}^{\mathrm{HAR}}=\left[\frac{1}{M} \sum_{m=1}^{M}\left[\widehat{G}^{[m]}\right]^{-1}\right]^{-1} .
$$

Since $\left[\widehat{G}^{[m]}\right]^{-1}=\mathbf{U}^{[m]}\left[\mathbf{Y}^{[m]}\right]^{-1}$, this actually corresponds to calculating the arithmetic mean of the output-input behavior, and then taking the inverse $\left(n_{y}=\right.$ $n_{u}$ is required). The output DFT matrices, $\mathbf{Y}^{[m]}, m=1, \ldots, M$, are then inverted, compared to inverting the input DFT matrices when using the ARI estimator (7). Note that the HAR estimator also uses the matrix function approach.

In this paper, these estimators will be evaluated experimentally. For a theoretical discussion about the estimators and their properties, see the included references in this paper as well as the discussion in Section 5.3.

\section{Excitation Signals}

In general the choice of excitation signal offers a large freedom in terms of frequency contents, magnitude, and so on, as long as the matrix $\mathbf{U}\left(\omega_{k}\right)$ has rank $n_{u}$. In this paper, the orthogonal random phase multisine signal will be used. This signal has been suggested in Dobrowiecki et al. (2005) and Dobrowiecki and Schoukens $(2005,2007)$ as input in the open-loop case to minimize the FRF uncertainty, given input amplitude constraints. Here, the orthogonal multisine signal will be used as reference signal, which corresponds to an optimal experiment design given output amplitude constraints. Each block in (5) is then given by

$$
\mathbf{R}^{[m]}\left(\omega_{k}\right)=\mathbf{R}_{\mathrm{diag}}^{[m]}\left(\omega_{k}\right) \mathbf{T}, \quad m=1, \ldots, M,
$$


with

$$
\mathbf{R}_{\text {diag }}^{[m]}\left(\omega_{k}\right)=\operatorname{diag}\left\{R_{l}^{[m]}\left(\omega_{k}\right)\right\}_{l=1}^{n_{y}},
$$

a diagonal matrix where each $R_{l}^{[m]}\left(\omega_{k}\right)$ is a random phase multisine signal, and $\mathbf{T}$ is an orthogonal matrix. A scalar random phase multisine signal $r(t)$ can be written as

$$
r(t)=\sum_{k=1}^{N_{f}} A_{k} \cos \left(\omega_{k} t+\phi_{k}\right),
$$

with amplitudes $A_{k}$, frequencies $\omega_{k}$ chosen from the grid $\left\{\frac{2 \pi l}{N_{P} T_{s}}, l=1, \ldots, N_{P} / 2-\right.$ 1\} $\left(N_{P}\right.$ even), and random phases $\phi_{k}$ uniformly distributed on the interval $[0,2 \pi)$.

The optimal matrix $\mathbf{T}$, with constraints $\left|\mathbf{T}_{i l}\right| \leq 1$, is given by (Dobrowiecki and Schoukens, 2007)

$$
\mathbf{T}_{i l}=e^{\frac{2 \pi j}{n_{u}}(i-1)(l-1)} .
$$

The number of frequencies as well as the amplitude spectrum will affect the quality of the FRF estimate. Using too many frequencies will give a low SNR, which increases both the bias and the variance in the estimate. The FRF estimate can be improved by averaging over multiple blocks and/or periods. The covariance matrix can then also be estimated.

For a linear system, averaging over different periods is sufficient, whereas for a nonlinear system, it is essential to average over blocks where $\mathbf{R}_{\text {diag }}$ in each block should have different realizations of the random phases. The reason is that nonlinearities otherwise will distort the estimate and give a too low uncertainty estimate. To reduce these nonlinear distortions, one should also excite only odd frequencies (odd $l$ in the frequency grid for (14)). For details, see Dobrowiecki and Schoukens (2007) and Pintelon and Schoukens (2001, Chap. 3). For the industrial robot, the nonlinearities cause large distortions (Wernholt and Gunnarsson, 2006). Averaging over multiple blocks is therefore important, so the EIV estimator is not applicable here.

\section{Experimental Results}

\subsection{Measurement Data}

As excitation, the orthogonal random phase multisine signal is used as motor speed reference $r$ with period time $T_{0}=N_{P} T_{s}=10 \mathrm{~s}$. A flat amplitude spectrum is used for $100 \log$-spaced odd frequencies between 1 and $60 \mathrm{~Hz}$ (i.e., the grid $1.1,1.3, \ldots, 59.9 \mathrm{~Hz}$ ). To reduce nonlinear effects, $M=100$ blocks with different realizations of the random phases are used. The magnitude of $\mathbf{R}_{\text {diag }}^{[\mathrm{m}]}$ is the same in all blocks. The commanded input $u$ and measured output $y$ are both sampled at $f_{s}=1 / T_{s}=2 \mathrm{kHz}$.

To reduce the effect of static friction, it is common to use an excitation signal that avoids zero velocity as much as possible. Therefore the reference is given by $r(t)=r_{m s}(t)+r_{s}(t)$, with $r_{m s}(t)$ the multisine signal and $r_{s}(t)$ a single sinusoid with frequency $0.7 \mathrm{~Hz}$. The amplitude of $r_{s}(t)$ is selected sufficiently larger than $\max _{t}\left|r_{m s}(t)\right|$ to avoid passing through zero velocity due to the multisine. Reducing the effect of static friction is really important for this type of system, 
as has been pointed out in Wernholt and Gunnarsson (2006). A drawback is that the sinusoid introduces large errors at a few of its overtones due to nonlinearities in the system. The first five overtones, $2.1,3.5,4.9,6.3,7.7 \mathrm{~Hz}$, are therefore removed in the resulting FRF estimate.

The FRF estimates turn out to have fairly large errors at low frequencies. Therefore, 10 additional blocks of data are collected using only four frequencies in the multisine $(1.1,2.7,4.3,5.9 \mathrm{~Hz})$. This improves the SNR, which in turn will reduce the error at these frequencies.

In the figures, the FRF estimates from motor torque to motor acceleration are plotted. That avoids the integrator $(-20 \mathrm{~dB} /$ decade $)$ such that resonances appear more clearly.

\subsection{Evaluation of FRF Estimators}

The five estimators from Section $3\left(\mathrm{H}_{1}\right.$, JIO, ARI, HAR, LOG) will now be used for FRF estimation. Since the true system is unknown, we cannot calculate the bias of the estimators. The assessment of the FRF estimates will therefore be partly based on experience from robot experts, as well as knowledge about the system and theoretical results.

The magnitude and phase of the resulting estimates, using 100 blocks of measurement data, are shown in Figure 3. The magnitude estimates agree quite well for frequencies above $5 \mathrm{~Hz}$, except for the $\mathrm{H}_{1}$ estimator which tends to give much lower values. For low frequencies $(1-5 \mathrm{~Hz})$, the estimates fluctuate quite much. By comparing the estimates with the ones using 10 blocks with four frequencies (circles in Figure 3), it is evident that the $\mathrm{JIO}$ and $\mathrm{H}_{1}$ estimators give large errors there. The estimators based on nonlinear averaging, HAR and LOG, give almost the same estimate and agree very well with the estimates using four frequencies. The ARI estimator works much better than JIO and $\mathrm{H}_{1}$, but does not match the performance of HAR and LOG (compare, e.g., element $(2,3)$ ). Considering the phase estimates, all five estimators agree remarkably well for frequencies above $5 \mathrm{~Hz}$. For low frequencies, the HAR and LOG estimators give much better estimates than the others.

In the simulation study in Wernholt and Gunnarsson (2007) it was noted that the uncertainties in nonparametric FRF estimates usually are non-symmetric, even if the true system is symmetric. This can be seen in Figure 3 as well, where one standard deviation of the ARI estimator is included. Compare, e.g., elements $(1,2)$ and $(2,1)$, or elements $(2,3)$ and $(3,2)$. For small elements, the relative error is also larger, which can be seen by comparing elements $(1,1)$ and $(2,1)$. These effects are inherent in all the studied estimation methods. From the standard deviation, it is also evident that the classical estimators $\left(\mathrm{H}_{1}, \mathrm{JIO}\right.$, and ARI) cannot handle a low SNR (at low frequencies and resonances) as good as the estimators based on nonlinear averaging techniques (HAR and LOG).

The estimates in Figure 3 are very clear and accurate, but measuring 100 blocks takes almost 3 hours in our experimental setup (approximately 1 minute per block of effective measurements, plus additional time for saving data, etc.). It is therefore interesting to study the quality of the estimates using less data.

Figure 4 shows the estimates using only 5 blocks, where LOG using 100 blocks is included for comparison. For low frequencies, all estimators give biased estimates in element $(2,1)$. Otherwise, the same comments as for Figure 3 are valid here as well. To be noted is that ARI fluctuates quite much at the 

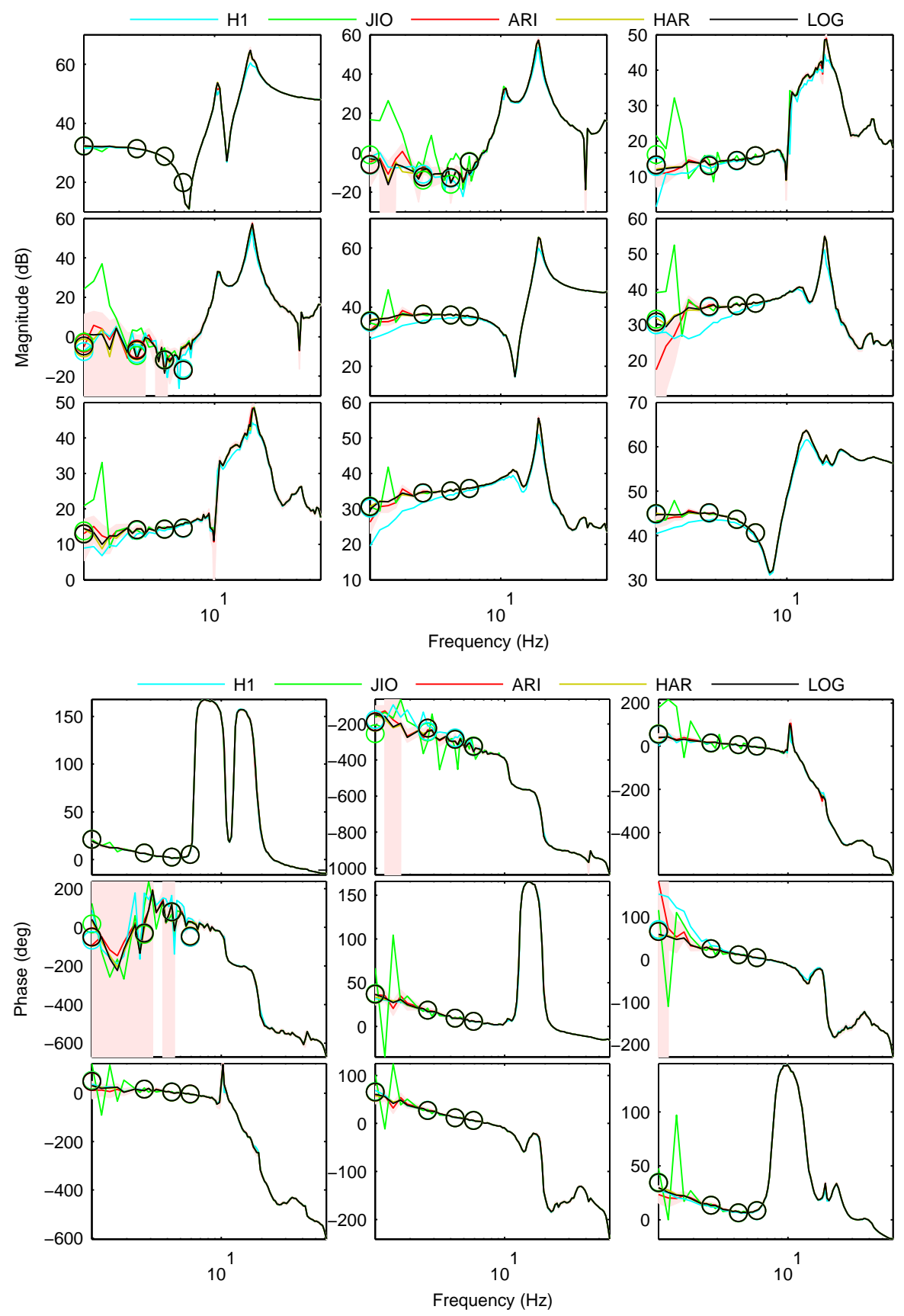

Figure 3: Estimated FRFs using 100 blocks of data. ARI is plotted with one standard deviation (shaded). Circles: estimates using 10 blocks with four frequencies. 

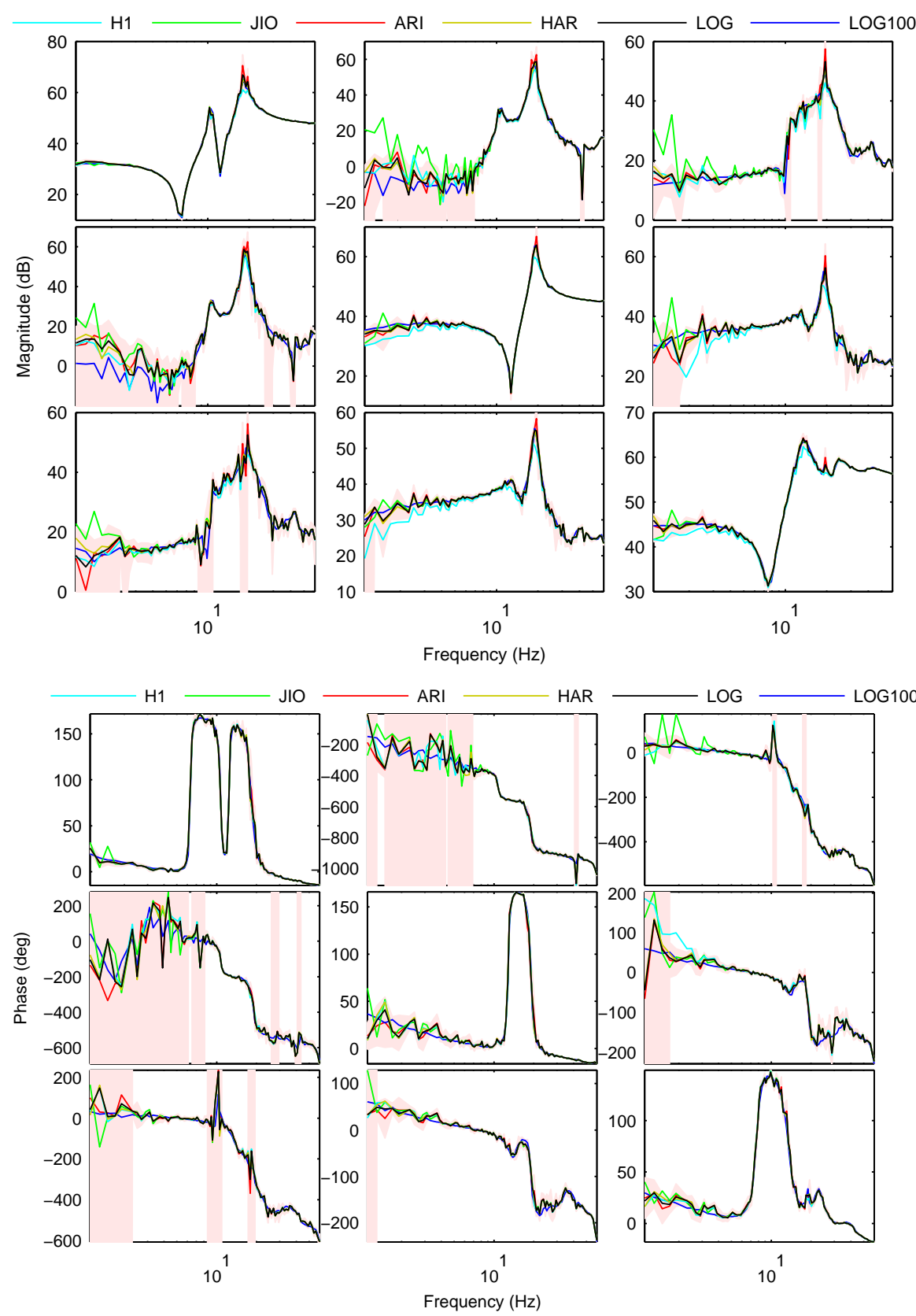

Figure 4: Estimated FRFs using 5 blocks of data. For comparison is LOG with 100 blocks included as well. ARI is plotted with one standard deviation (shaded). 

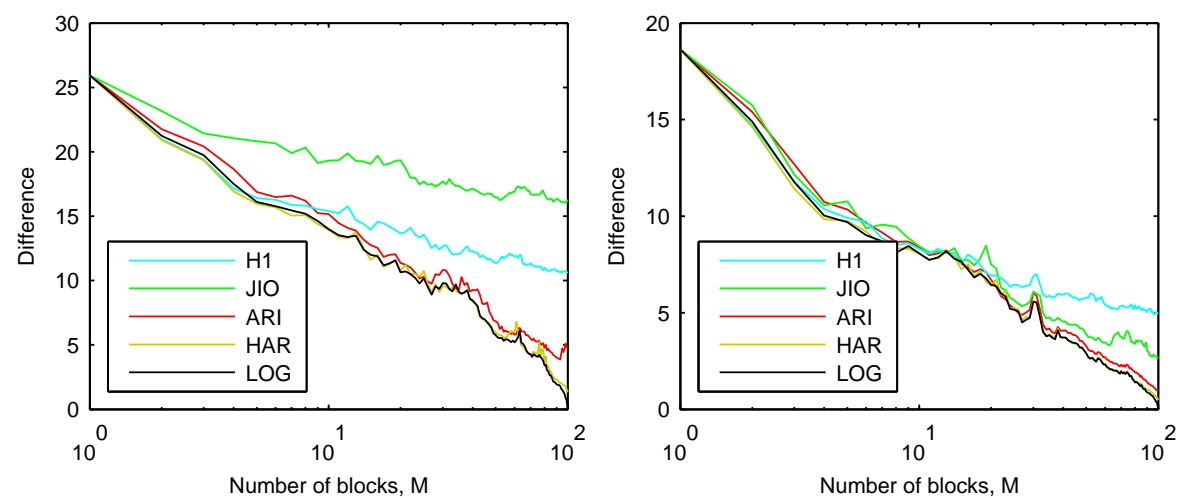

Figure 5: Difference $c_{l o g}\left(\widehat{G}_{100}^{\mathrm{LOG}}, \widehat{G}_{M}\right)$ for $1-60 \mathrm{~Hz}$ (left) and $5-60 \mathrm{~Hz}$ (right) as a function of the number of blocks, $M . \widehat{G}_{M}$ is replaced by each of the five estimators.

resonances. Since the reference signal has a flat amplitude spectrum, the input is small at the resonances. The fluctuations are therefore probably caused by inverting a matrix that is quite sensitive to noise.

Next, we will compare the different estimators as a function of the number of blocks. Let $\widehat{G}_{M}$ denote the FRF estimate calculated from $M$ blocks of data. Consider now the difference between $\widehat{G}_{100}^{\mathrm{LOG}}$ (the LOG estimate in Figure 3 ) and the estimates $\widehat{G}_{M}^{\mathrm{H}_{1}}, \widehat{G}_{M}^{\mathrm{JIO}}, \widehat{G}_{M}^{\mathrm{ARI}}, \widehat{G}_{M}^{\mathrm{HAR}}$, and $\widehat{G}_{M}^{\mathrm{LOG}}$, where the number of blocks, $M$, will vary between 1 and 100 . To measure the difference between two estimates, the following cost is used

$$
c_{l o g}\left(G^{1}, G^{2}\right)=\left(\sum_{i, j, k}\left|\log G_{i j}^{1}\left(\omega_{k}\right)-\log G_{i j}^{2}\left(\omega_{k}\right)\right|^{2}\right)^{1 / 2} .
$$

In addition, phase wrapping problems in the complex logarithm are handled to ensure that $\left|\arg G_{i j}^{1}\left(\omega_{k}\right)-\arg G_{i j}^{2}\left(\omega_{k}\right)\right| \leq \pi$. The difference between $\widehat{G}_{100}^{\mathrm{LOG}}$ and the other estimates can be seen in Figure 5 .

First, note that all estimators coincide for $M=1$. When $M$ is increased, so is the difference between the estimators. The most interesting thing to note from Figure 5 might be that HAR and LOG give better results than the other estimators for all $M>1$. HAR might be slightly better than LOG for small $M$, but they otherwise give approximately the same performance. What might be surprising, given the previous results in Figures 3 and 4 , is that $\mathrm{H}_{1}$ gives almost the same cost as LOG and HAR for low values of $M$. The explanation can be found in Figure 4 where all estimates differ from $\widehat{G}_{100}^{\mathrm{LOG}}$ for low frequencies. Another interesting thing to note is that ARI gives a larger cost than $\mathrm{H}_{1}$ when $M \leq 10$. The main reason is the large errors at the resonances for ARI. JIO gives the worst overall performance, mainly due to the errors at low frequencies. Considering only the interval $5-60 \mathrm{~Hz}$ in Figure 5, JIO and ARI give approximately the same cost for $M \leq 30$, except that the cost for JIO fluctuates more.

Since we are comparing the estimates with $\widehat{G}_{100}^{\mathrm{LOG}}$, the fact that LOG and 
HAR are the best comes as no surprise when $M$ is close to 100 . However, this is also the case if we compare with $\widehat{G}_{100}^{\mathrm{ARI}}$ (for $M \leq 50$ ) or some of the other estimators. The previous conclusion that LOG and HAR are the best estimators therefore holds also in this comparison. Finally, this section is ended by Figure 6, where estimates using LOG for some values of $M$, including $M=1$, are given. As can be seen, the fluctuations are fairly large when using only one block of data.

\subsection{Discussion}

From theoretical studies, as well as experimental experience, it is known that the bias depends on the SNR, such that the bias is reduced when the SNR is increased. The data set with 10 blocks and four frequencies can therefore be considered to give a smaller bias. This can also be seen in Figure 3 where all five estimators give approximately the same estimate. Since we are dealing with linear approximations of a nonlinear system, a comparison of estimates using different types of excitation signals should however be done with some care. The best linear approximation might be different when changing the amplitude spectrum and/or the signal shape. This can, e.g., be seen in Wernholt and Gunnarsson (2006) where estimates are compared when using different excitation amplitudes as well as with and without a filtered square wave (to reduce the effect of static friction).

The $\mathrm{H}_{1}$, ARI, and JIO estimators have been analyzed in, e.g., Wernholt and Gunnarsson (2007), Pintelon and Schoukens (2001) and Verboven (2002). The $\mathrm{H}_{1}$ and ARI estimators are known to give biased estimates for closed-loop data. The bias of the $\mathrm{H}_{1}$ estimator is much larger, except for small SNRs where the ARI estimator deteriorates. The JIO estimator is asymptotically unbiased $(M \rightarrow \infty)$ and is therefore expected to give the best performance when the number of blocks increases. This is also the conclusion in Wernholt and Gunnarsson (2007), where the JIO, ARI, and $\mathrm{H}_{1}$ estimators are compared in a simulation study with a similar setup as in this paper, but with a linear robot model. In our experimental results, the JIO estimator does not seem to perform that well, at least not if the low-frequency behavior is considered.

A limitation with the JIO estimator, besides that an additional signal is needed, is that the whole measurement setup must be linear, including the controller. This is due to the fact that $\widehat{G}^{\mathrm{JIO}}$ actually is based on $\mathrm{H}_{1}$ estimates of the FRFs from $r$ to $y, \widehat{G}_{r y}$, and $r$ to $u, \widehat{G}_{r u}$, as $\widehat{G}^{\mathrm{JIO}}=\widehat{G}_{r y} \widehat{G}_{r u}^{-1}$. Both these FRFs incorporate the whole measurement setup, including the controller. In our application, the controller, e.g., has a saturation to ensure that the motors are not overloaded. The other methods considered in this paper are only affected by nonlinearities in the input-output system.

The properties of the LOG and HAR estimators have been analyzed in e.g., Guillaume et al. (1992), Guillaume (1998), and Pintelon and Schoukens (2001). The HAR estimator is particularly useful when the input SNR is low since the input DFT matrix $\mathbf{U}^{[m]}$ is not inverted. In our setup we are using the orthogonal multisine signal as reference $\mathbf{R}^{[m]}$, so the output $\mathbf{Y}^{[m]}$ will be approximately orthogonal as well, or at least well-conditioned. The input $\mathbf{U}^{[m]}$, on the other hand, can be quite ill-conditioned for certain frequencies (e.g., close to resonances, or for low frequencies since we are using a flat speed reference signal). The HAR estimator is therefore well suited for our setup. The LOG estimator is 

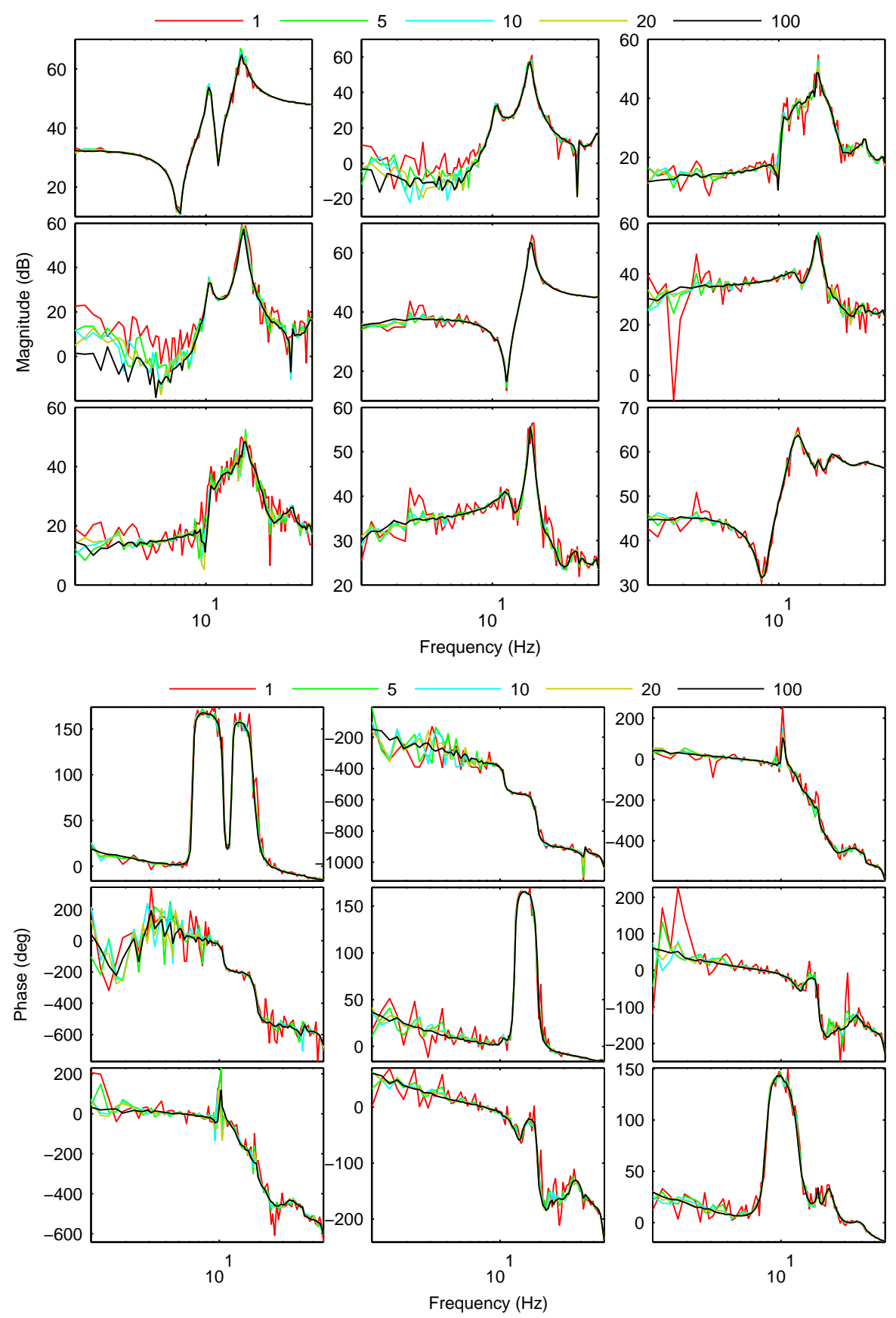

Figure 6: Estimated FRFs using the LOG estimator for different number of blocks. 
inherently robust to outliers since the logarithmic function attenuates the effect of occasional large errors. For the classical estimators, a large number of blocks are needed to average out such effects. This can, e.g., be noted with the ARI estimator at the resonances in Figure 4.

As was mentioned in Section 5.1, it is important to compensate for the static friction. Using the single sinusoid caused large errors at some of its overtones due to nonlinearities in the system. This might depend on the fact that its phase is the same in all experiments. Different ways of reducing the effects of static friction would therefore be interesting to study.

As future work remains a thorough simulation study using a realistic nonlinear robot model where friction as well as other nonlinear effects in actuators and sensors are added, such as torque ripple (Gutt et al., 1996) and resolver ripple (Hanselman, 1990). The bias can then be studied for the different estimators as a function of the excitation signal and the nonlinearities.

\section{Conclusion}

This paper has dealt with nonparametric multivariable FRF estimation using closed-loop data from an industrial robot. Three classical estimators $\left(\mathrm{H}_{1}\right.$, JIO, ARI) and two estimators based on nonlinear averaging techniques (HAR, LOG) have been compared. The orthogonal random phase multisine signal has been used, where the number of experiments have been varied to see how the estimators depend on the amount of data. The estimators based on nonlinear averaging give the best results, followed by the arithmetic mean estimator, which gives a slightly larger bias. The joint input-output estimator, which is asymptotically unbiased in theory, turns out to give large bias errors for low frequencies. Finally, the $\mathrm{H}_{1}$ estimator gives the largest bias for all frequencies.

\section{References}

E. Berglund and G. E. Hovland. Automatic elasticity tuning of industrial robot manipulators. In Proc. 39th IEEE Conference on Decision and Control, pages 5091-5096, Sydney, Australia, December 2000.

R. Cobb and L. Mitchell. Estimates of variance and confidence bands for the three-channel frequency response function estimator. International Journal of Analytical and Experimental Modal Analysis, 5(3):185-194, July 1990.

T. Dobrowiecki and J. Schoukens. Measuring linear approximation to weakly nonlinear MIMO systems. In Proc. 16th IFAC World Congress, Prague, Czech Republic, July 2005.

T. Dobrowiecki and J. Schoukens. Measuring a linear approximation to weakly nonlinear MIMO systems. Automatica, 43(10):1737-1751, October 2007.

T. Dobrowiecki, J. Schoukens, and P. Guillaume. Optimized excitation signals for MIMO frequency response function measurments systems. In Proc. 22nd IEEE Instrumentation and Measurement Technology Conference, pages 18721877, June 2005. 
P. Guillaume. Frequency response measurements of multivariable systems using nonlinear averaging techniques. IEEE Transactions on Instrumentation and Measurement, 47(3):796-800, June 1998.

P. Guillaume, R. Pintelon, and R. Schoukens. Nonparametric frequency response function estimators based on nonlinear averaging techniques. IEEE Transactions on Instrumentation and Measurement, 41(6):739-746, December 1992.

P. Guillaume, R. Pintelon, and J. Schoukens. Accurate estimation of multivariable frequency response functions. In Proc. 13th IFAC World Congress, pages 423-428, San Francisco, California, 1996.

H.-J. Gutt, F. D. Scholl, and J. Blattner. High precision servo drives with DSPbased torque ripple reduction. In Proc. IEEE AFRICON, 1996, volume 2, pages 632-637, September 1996.

D. Hanselman. Resolver signal requirements for high accuracy resolver-to-digital conversion. IEEE Transactions on Industrial Electronics, 37(6):556-561, December 1990.

F. Khorrami, S. Jain, and A. Tzes. Experimental results on adaptive nonlinear control and input preshaping for multi-link flexible manipulators. Automatica, 31(1):83-97, January 1995.

L. Ljung. System Identification: Theory for the User. Prentice Hall, Upper Saddle River, New Jersey, USA, 2nd edition, 1999.

J. Öhr, S. Moberg, E. Wernholt, S. Hanssen, J. Pettersson, S. Persson, and S. Sander-Tavallaey. Identification of flexibility parameters of 6 -axis industrial manipulator models. In Proc. ISMA2006 International Conference on Noise and Vibration Engineering, pages 3305-3314, Leuven, Belgium, September 2006 .

R. Pintelon and J. Schoukens. System identification: a frequency domain approach. IEEE Press, New York, 2001.

R. Pintelon and J. Schoukens. Measurement of frequency response functions using periodic excitations, corrupted by correlated input/output errors. IEEE Transactions on Instrumentation and Measurement, 50(6):1753-1760, December 2001.

J. Schoukens, R. Pintelon, T. Dobrowiecki, and Y. Rolain. Identification of linear systems with nonlinear distortions. Automatica, 41(3):491-504, March 2005 .

P. Verboven. Frequency-domain system identification for modal analysis. $\mathrm{PhD}$ thesis, Vrije Universiteit Brussel, Belgium, May 2002.

P. E. Wellstead. Nonparametric methods of system identification. Automatica, 17(1):55-69, January 1981

E. Wernholt and S. Gunnarsson. Detection and estimation of nonlinear distortions in industrial robots. In Proc. 23rd IEEE Instumentation and Measurement Technology Conference, pages 1913-1918, Sorrento, Italy, April 2006. 
E. Wernholt and S. Gunnarsson. Analysis of methods for multivariable frequency response function estimation in closed loop. In 46th IEEE Conference on Decision and Control, New Orleans, Louisiana, December 2007. Accepted for publication.

E. Wernholt and S. Moberg. Frequency-domain gray-box identification of industrial robots. Technical Report LiTH-ISY-R-2826, Department of Electrical Engineering, Linköping University, SE-581 83 Linköping, Sweden, October 2007. Submitted to the 17th IFAC World Congress, Seoul, Korea. 


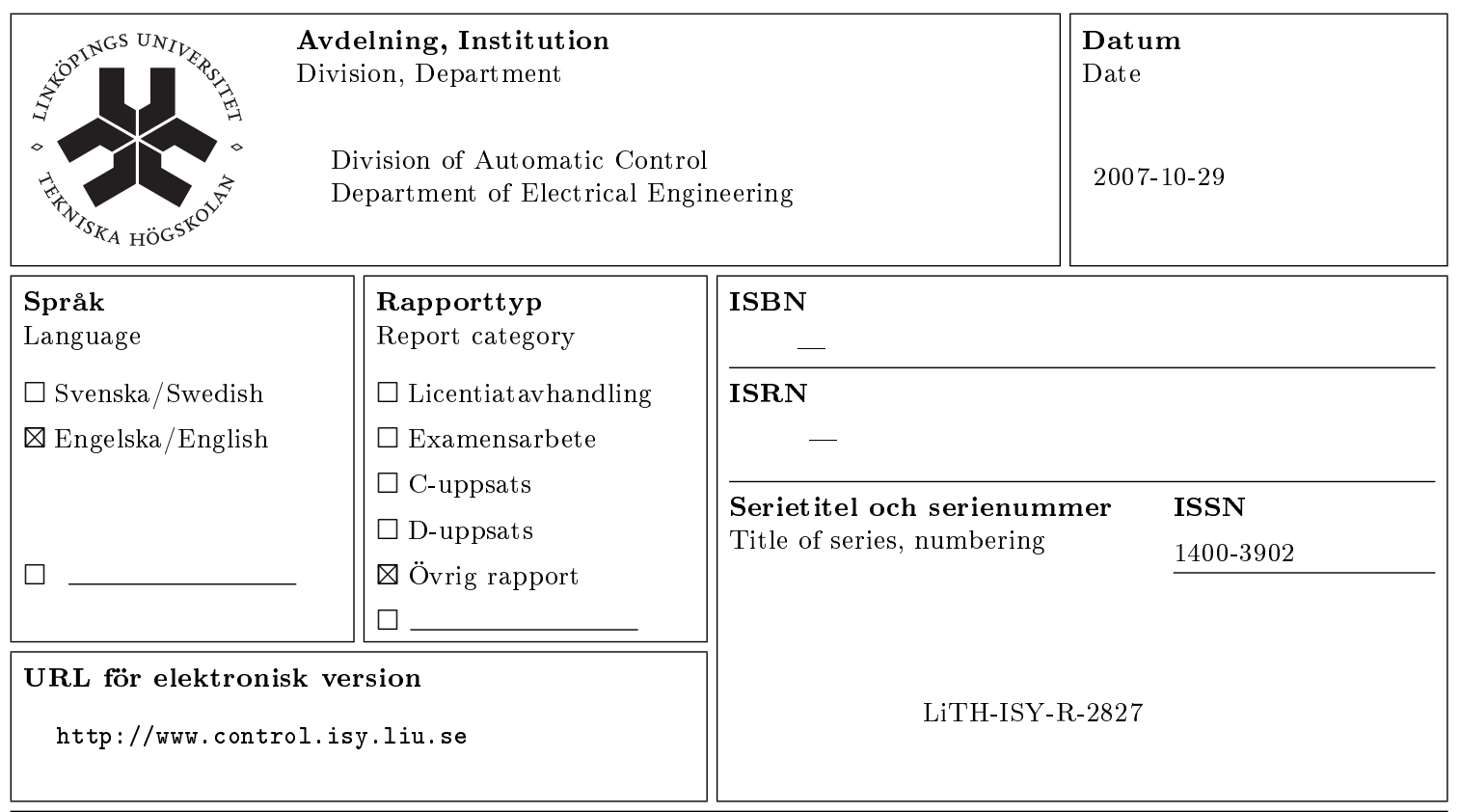

Titel Experimental Comparison of Methods for Multivariable Frequency Response Function EstiTitle mation

Författare Erik Wernholt, Stig Moberg

Author

\section{Sammanfattning}

Abstract

Nonparametric estimation methods for the multivariable frequency response function are experimentally evaluated using closed-loop data from an industrial robot. Three classical estimators $\left(\mathrm{H}_{1}\right.$, joint input-output, arithmetic mean) and two estimators based on nonlinear averaging techniques (harmonic mean, geometric/logarithmic mean) are considered. The estimators based on nonlinear averaging give the best results, followed by the arithmetic mean estimator, which gives a slightly larger bias. The joint input-output estimator, which is asymptotically unbiased in theory, turns out to give large bias errors for low frequencies. Finally, the $\mathrm{H}_{1}$ estimator gives the largest bias for all frequencies. 